\title{
Comparison of Expected Crash and Injury Reduction from Production Forward Collision and Lane Departure Warning Systems
}

\author{
Online Supplemental Appendix \\ Kristofer D. Kusano and Hampton C. Gabler \\ Virginia Tech
}

Corresponding Author:

Kristofer D. Kusano

440 Kelly Hall, 325 Stanger St

Blacksburg, VA 24061

kusano@vt.edu

Submitted to Traffic Injury Prevention

(AAAM 2015 Special Edition)

June 16, 2015 


\section{Methodology Overview}

Figure 1 shows the benefits estimate framework proposed for the Advanced Crash Avoidance Trechnologies (ACAT) program (Carter et al. 2009; Funke et al. 2010).

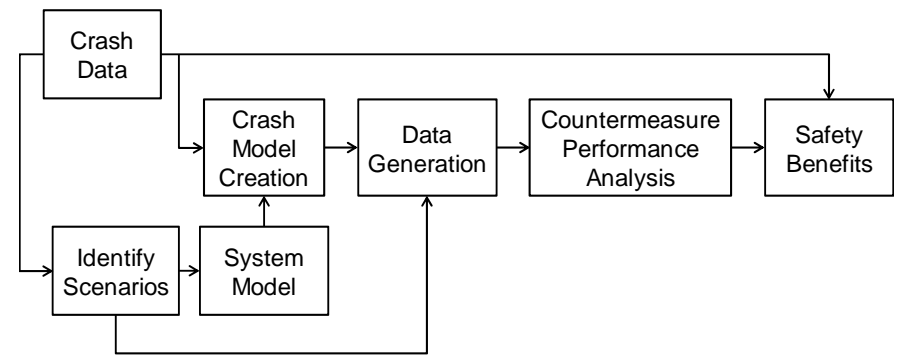

Figure 1. Safety Benefits Analysis Framework.

Figure 2 shows the general organization of the benefits estimates methods used for this study. Additional details of these past studies are provided in Kusano and Gabler (2012) for the FCW model and Kusano et al. (2014) for the LDW model.

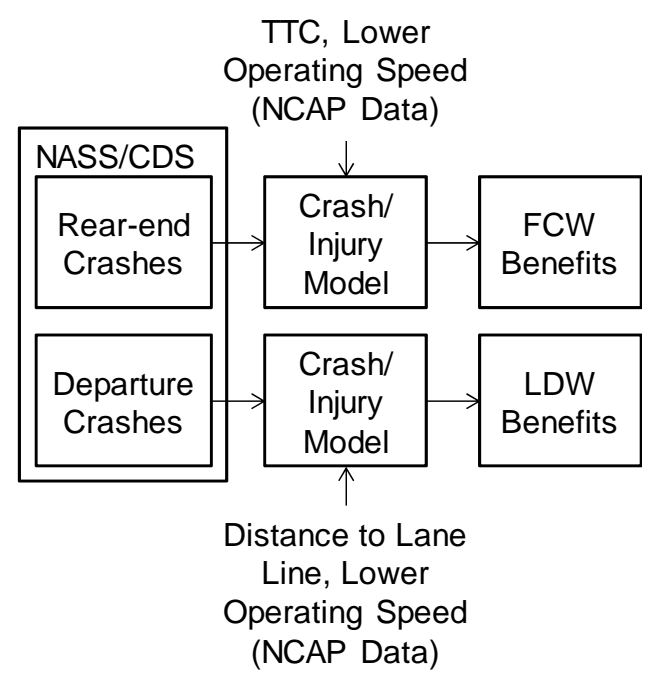

Figure 2. FCW and LDW Benefits Estimate Models.

Table 1 lists the abbreviations and their definitions used in this paper. The abbreviations are ordered as they first appear in the text. 
Table 1. List of Abbreviations used in this Paper.

\begin{tabular}{|l|l|}
\hline Abbreviation & Definition \\
\hline FCW & Forward Collision Warning \\
\hline AEB & Automated Emergency Braking \\
\hline LDW & Lane Departure Warning \\
\hline LDP & Lane Departure Prevention \\
\hline NASS/CDS & $\begin{array}{l}\text { National Automotive Sampling } \\
\text { System, Crashworthiness Data } \\
\text { System }\end{array}$ \\
\hline ACAT & $\begin{array}{l}\text { Advanced Crash Avoidance } \\
\text { Technologies }\end{array}$ \\
\hline TTC & Time to Collision \\
\hline MAIS & Maximum Abbreviated Injury Scale \\
\hline
\end{tabular}

\section{New Car Assessment Program Confirmation Test Description}

The U.S. NCAP consumer ratings include the presence of FCW or LDW. To verify the claim that a vehicle has FCW and/or LDW, a test track procedure is used to determine if the function of the system meets NCAP specifications. These test track procedures are called confirmation tests (NHTSA 2013a; NHTSA 2013b). This section summarizes the test procedures for the FCW and LDW confirmation tests used to construct models of each production system for this study.

The FCW confirmation test matrix is summarized in Table 2. The subject vehicle is driven at a constant speed of $45 \mathrm{mph}$ (72.4 kph) approaching a lead vehicle that is either stopped, braking, or moving at a lesser speed. For the lead vehicle braking trial, the lead vehicle begins to brake at $0.3 \mathrm{~g}$ when

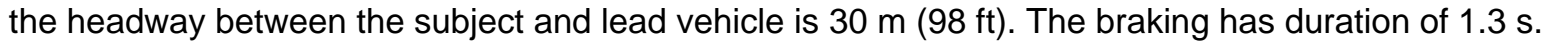
Between 7 and 5 trials are performed for each scenario. The test is a success if the subject vehicle delivers a warning before a given TTC threshold. TTC is calculated as the instantaneous ratio of the range to range rate and does not include an acceleration term.

Table 2. NCAP Confirmation Test Conditions for FCW

\begin{tabular}{|l|l|l|l|}
\hline $\begin{array}{l}\text { Lead Vehicle } \\
\text { Scenario }\end{array}$ & $\begin{array}{l}\text { Subject } \\
\text { Vehicle } \\
\text { Speed } \\
\text { (mph) }\end{array}$ & $\begin{array}{l}\text { Lead } \\
\text { Vehicle } \\
\text { Speed } \\
\text { (mph) }\end{array}$ & $\begin{array}{l}\text { TTC at } \\
\text { Least } \\
\text { (s) }\end{array}$ \\
\hline Stopped & 45 & 0 & 2.1 \\
\hline Decelerating & 45 & $\begin{array}{l}45 \mathrm{mph}, \\
0.3 \mathrm{~g} \\
\text { braking }\end{array}$ & 2.4 \\
\hline Moving & 45 & 20 & 2.0 \\
\hline
\end{tabular}


Figure 3 shows the test configuration for the LDW confirmation test. The subject vehicle is driven at a constant speed of $45 \mathrm{mph}(72 \mathrm{kph})$ on a paved surface with a single lane line painted on the side of departure. The test begins after the vehicle passes through a set of 4 pylons. After the pylons, a test driver steers the vehicle over the lane line at a lateral speed between $0.1 \mathrm{~m} / \mathrm{s}$ and $0.6 \mathrm{~m} / \mathrm{s}$. The test is a success if the subject vehicle delivers a warning when the outside of the vehicle's leading wheel is between $0.75 \mathrm{~m}$ before crossing the lane line and $0.3 \mathrm{~m}$ after crossing the lane line. The test matrix is composed of standard solid yellow, dashed white, and "Botts dots" raised pavement markers. Each marking is tested departing to the left and right for a total of 6 test conditions. Each test condition is repeated 5 times.

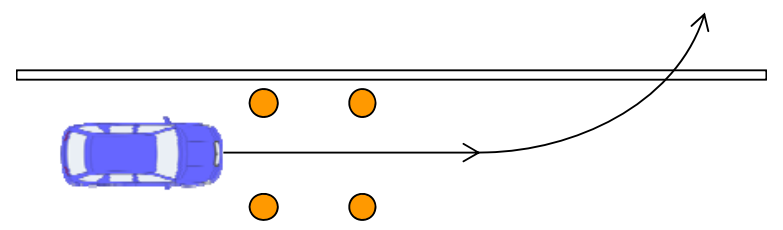

Figure 3. New Car Assessment Program LDW Confirmation Test Procedure.

\section{Methodology for Estimating Fleetwide Safety Benefits}

Both methodologies are based upon crash data extracted from the National Automotive Sampling System Crashworthiness Data System (NASS/CDS). NASS/CDS is a nationally representative data collection effort funded by the U.S. Department of Transportation. Approximately 4,000-5,000 crashes are investigated throughout the U.S. each year. Crash investigation teams photograph and diagram the crash scene, measure vehicle damage, perform crash reconstructions, and collect medical records. This detailed investigation makes NASS/CDS a rich data source for reconstructing and modeling crashes as if they occurred with a crash avoidance system present. The NASS/CDS database includes sampling weights that make the sample nationally representative. The sample weights are developed by the NASS/CDS designers and indicate how many similar collisions occurred but were not investigated during the study period. In this study, all results and benefits estimates use the sampling weights provided in NASS/CDS.

The crash scenarios that would most likely be mitigated by FCW are rear-end collisions and the scenario most likely mitigated by LDW are single vehicle road departure crashes. The benefit estimate model for FCW estimated the number of crashes and seriously injured drivers that could have been prevented if all vehicles involved in rear-end collisions had FCW (Kusano and Gabler 2012). The benefit estimate model for LDW estimated benefits if all vehicles involved in drift out of lane, single vehicle road departure crashes were equipped with LDW (Kusano et al. 2014a). Rear-end and departure crashes were extracted from NASS/CDS using previously developed pre-crash scenarios (Kusano and Gabler 2014b). The pre-crash scenarios used pre-crash event, pre-crash vehicle movement, and accident type variables to group crashes into scenarios. For rear-end collisions, two vehicles must have impacted in a front to rear configuration with the striking vehicle not performing a traffic maneuver, e.g., turning, merging, or changing lanes. For departure crashes, a single vehicle must have departed its lane without maneuvers. 
Crashes coded with control loss prior to the lane departure or a collision with objects on the roadway were excluded.

Both the FCW and LDW benefits methodologies developed in previous studies simulated each crash multiple times to account for driver variability and road conditions not included in the database. Table 3 summarizes the driver reaction times used in the FCW and LDW models. The reaction time was defined as the time between the warning being delivered and the start of the vehicle's maneuver, braking only for FCW and steering only for LDW. Each reaction time chosen was assigned a weight based on how likely it was to occur in the population. The FCW reaction times were derived from a naturalistic study that measured brake reaction time of drivers on public roads (Sivak and Olson 1982). Data downloaded from event data recorders in NASS/CDS crashes indicated that approximately $17 \%$ of drivers in rear-end crashes do not apply the brakes before a collision (Kusano and Gabler 2012). The LDW reaction times were taken from a driving simulator experiment where drivers experienced LDW alerts. The experiments exposed occupants who were both aware and unaware of the LDW function prior to the experiment. Aware drivers had an average reaction time of $0.38 \mathrm{~s}$ while unaware drivers had a reaction time of $1.36 \mathrm{~s}$ (Suzuki and Jansson 2003). The probability of a driver being aware or unaware of the LDW system was assumed to be equal and both reaction times were assigned a weight of $50 \%$.

Table 3. Driver Reaction Time to Warning and Simulation Weights for FCW and LDW Models.

\begin{tabular}{|l|l|l|l|l|l|}
\hline & \multicolumn{2}{|c|}{ FCW } & & \multicolumn{2}{c|}{ LDW } \\
\hline Reaction & Time (s) & Weight (\%) & & Time (s) & Weight (\%) \\
\hline 1 & 0.57 & $20 \%$ & & 0.38 & $50 \%$ \\
\hline 2 & 1.07 & $43 \%$ & & 1.36 & $50 \%$ \\
\hline 3 & 1.48 & $20 \%$ & & & \\
\hline 4 & No input & $17 \%$ & & & \\
\hline
\end{tabular}

Variation in driver braking and road conditions were considered in the FCW benefits model. Because the level of braking in the NASS/CDS crashes was unknown, each crash that the NASS/CDS investigation indicated there was driver braking without FCW was simulated as having both an "early, weak" and "late, hard" braking response. The weak braking was assumed at a level of $0.2 \mathrm{~g}$ starting well before the FCW would have been delivered. The hard late braking was assumed at a level of $0.4 \mathrm{~g}$ starting at a time to collision of $0.4 \mathrm{~s}$ (Kusano and Gabler 2012). The braking deceleration in reaction to FCW was also $0.4 \mathrm{~g}$. The allowable braking deceleration was limited to $0.15 \mathrm{~g}$ on icy pavement, or $0.25 \mathrm{~g}$ on wet pavement with wheel lockup. Surface conditions and wheel lockup was extracted from NASS/CDS.

Real-world crash data such as NASS/CDS often lack detailed measurements of the roadway characteristics needed to simulate LDW applicable crashes. To account for these missing variables, first the NASS/CDS scene photographs were manually examined to determine the presence of lane markings, approximate shoulder width, and road curvature at the point of the first departure. Second, using a special subset of NASS/CDS crashes with supplemental crash reconstructions, regression models were developed that predicted the departure speed, departure angle, and radius of curvature in the crash using 
NASS/CDS variables as predictors. These regression models were then applied to each NASS/CDS in the simulation case set to generate initial conditions. Because most LDW systems use camera systems to detect lane boundaries, in the model LDW had no benefit for crashes that did not have lane lines at the side of the first departure. Furthermore, in our model LDW did not have a benefit in crashes where there was no shoulder because the driver will have no additional time to steer back onto the road.

In order to evaluate the production FCW and LDW systems, parameters to model each system were extracted from the NCAP confirmation test data described in the following sections. The parameters of interest were warning activation (time to collision, TTC, for FCW and distance to lane cross for LDW) and the lower operating speed threshold of the systems.

The crash and injury models simulated the target crashes without and with the system present to determine the benefits. The injury reduction from the systems were developed using logistic regression on existing crash data. The FCW injury model was developed to predict the chance of a moderate to fatal injury, i.e., a maximum Abbreviated Injury Scale (MAIS) score of 2 or greater (MAIS2+). The predictors were change in velocity during the crash (delta- $V$ ), seat belt use, occupant age, and occupant sex. The LDW model predicted MAIS3+ injury with predictors of vehicle travel speed and seat belt status. MAIS2+ was selected as a threshold for rear-end crashes because MAIS3+ injuries are rare in this crash mode.

The benefit estimates produced in this study were made using several best-case assumptions and thus represent an upper limit of effectiveness. First, the models assumed $100 \%$ penetration in the fleet and that the drivers had the systems activated $100 \%$ of the time. Penetration of LDW and FCW into the fleet will take many years and may never reach a true $100 \%$ fitment rate. Additionally, some systems are turned off by default, requiring the driver to enable them, or can be disabled if the driver chooses to do so. Second, the models assumed the driver responded to the alerts and took the correct evasive action. Third, we also assumed that the alert timing derived from the NCAP confirmation test data was constant across all operating speeds. Some systems may alter alert timing based on other factors, e.g., vehicle speed.

The methodology for comparing production safety systems using NCAP confirmation test data was presented by the authors in a previous study (Kusano et al. 2014c). Since this previous study was performed, the amount of test data available for analysis has increased in size by almost 4 times, from 19 vehicles to 55 vehicles tested. Substantial enhancements have also been made to the LDW benefits estimate models (Kusano et al. 2014a) compared to a previous model used (Gabler et al. 2013). The previous study also did not investigate the effect of LDW operating speeds on system benefits. Finally, the previous study anonymized the vehicle makes and models since the focus of the paper was the methodology.

\section{Forward Collision Warning Characteristics}

Figure 4 shows the average TTC at warning delivery for NCAP tests in the lead vehicle stopped scenario. The red vertical line in the graph indicates the $2.1 \mathrm{~s}$ TTC threshold used to determine if a system passed the test. Three (3) vehicles had an average sound warning that was delivered after the NCAP threshold. 
All three of these vehicles had a visual warnings delivered on the instrument panel that met the test requirement and all three vehicles passed the NCAP confirmation test. Visual warnings on the instrument panel were not considered for our benefits estimate models. The presence of Automated Emergency Braking (AEB) was determined from the NCAP test reports. Out of the $46 \mathrm{FCW}$ tests conducted, 24 vehicles were also equipped with AEB. Vehicle names marked with an asterisk in Figure 4 had AEB equipped in addition to the FCW system.

Figure 5 shows the lower operating speed thresholds for the tested FCW systems. The vehicles are listed in the same order as in Figure 4. The systems ranged from near full operating range (1 or 5 $\mathrm{mph}$ threshold) to higher speed only (20 or $25 \mathrm{mph}$ ). The Mercedes Benz systems (2012 E350, ML350, CL550 and 2014 S550) were the only systems that listed a separate lower operating speed threshold for stationary vehicles ( $40 \mathrm{mph}$ ). The FCW system operates on moving objects at speeds above $4 \mathrm{mph}$ for the Mercedes Benz vehicles except the 2012 ML350, which had a threshold of $20 \mathrm{mph}$. Because the benefits estimate model only simulated lead vehicle stopped scenarios, the $40 \mathrm{mph}$ lower operating speed threshold was used for this study.

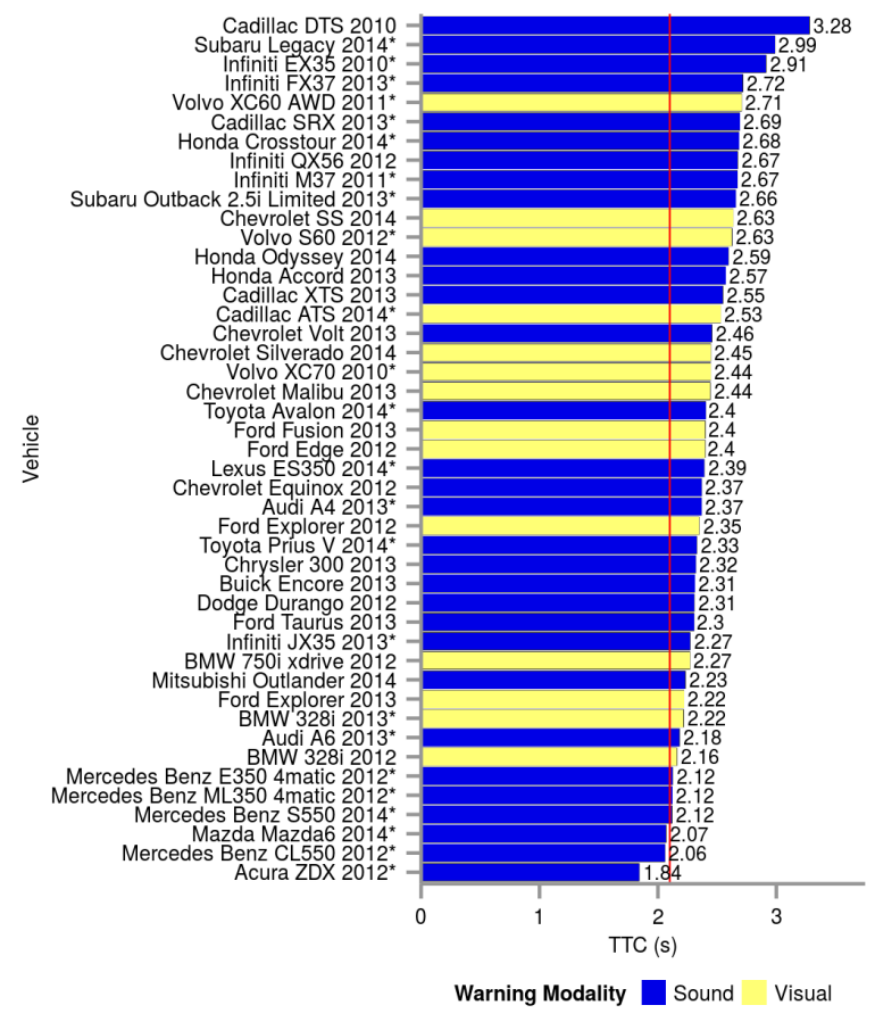

Figure 4. Average Warning Delivery TTC for Lead Vehicle Stopped FCW Tests. An asterisk indicates a vehicle that also had Automated Emergency Braking (AEB) equipped. 


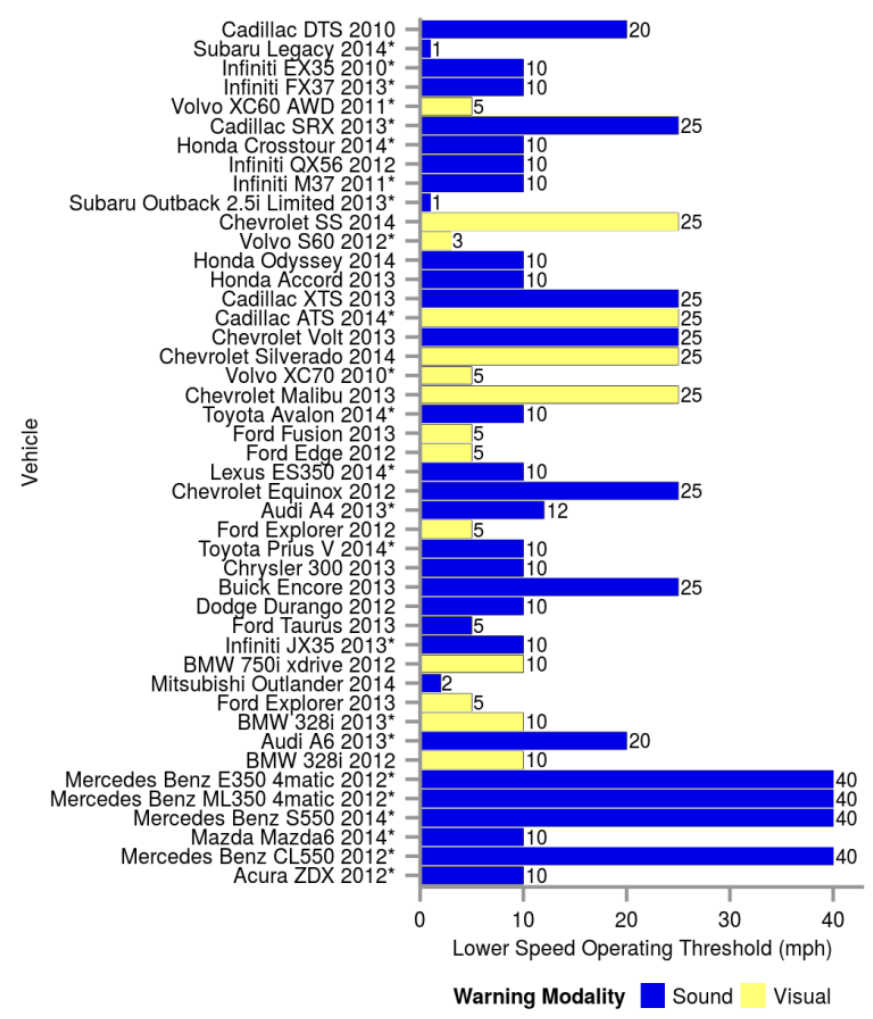

Figure 5. Lower Operating Speed Thresholds for FCW Systems. An asterisk indicates a vehicle that also had Automated Emergency Braking (AEB) equipped.

\section{Lane Departure Warning Characteristics}

Figure 6 shows the distance to the lane line that LDW was delivered by departure side and lane marking type. A positive distance indicates a warning that was delivered before crossing the lane line and a negative distance indicates a warning delivered after the vehicle crossed the lane line. Vehicles are ordered in descending order by average warning distance across all scenarios. Some vehicles were also equipped with a Lane Departure Prevention (LDP) system that could apply steering wheel torque or differentially apply the brakes to steer the vehicle back towards the center of the lane. Vehicles with LDP in addition to LDW are marked with an asterisk in Figure 6. Of the 40 vehicles tested, 10 had LDP available. Although there were differences between side/marking scenarios within vehicles, there does not appear to be a trend suggesting systems are designed to behave differently for different lane marking types. All of the warnings were well within the required NCAP thresholds of $0.75 \mathrm{~m}$ before the lane cross and $0.3 \mathrm{~m}$ after the lane cross. 


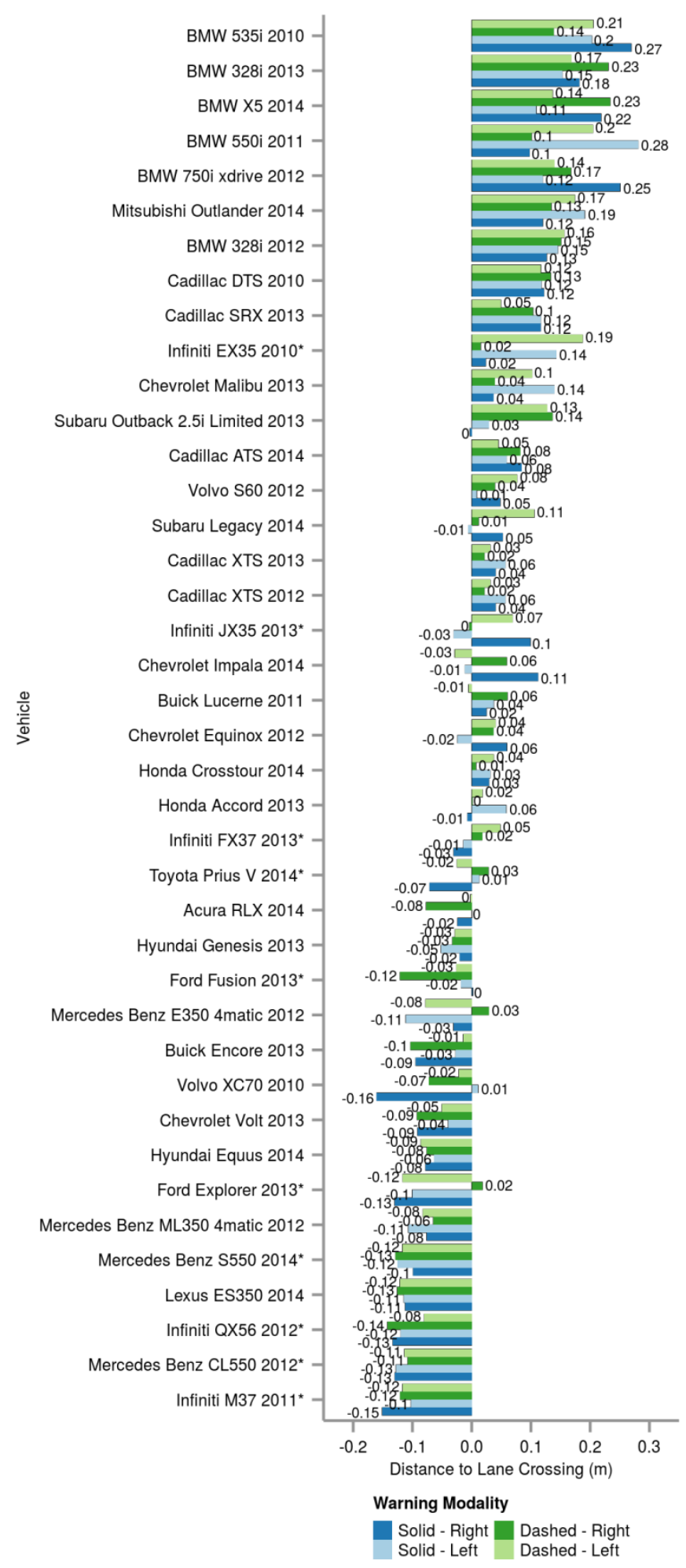

Figure 6. Distance to Lane Crossing at LDW Delivery. Positive distance is a warning delivered before departure, negative distance is warning delivered after departure. An asterisk indicates a vehicle that also had Automated Emergency Braking (AEB) equipped. 
Figure 7 shows the lower operating speed threshold for the LDW systems. The vehicles are in the same order as in Figure 6. The lower operating speeds for LDW systems are in general much higher than the thresholds for FCW.

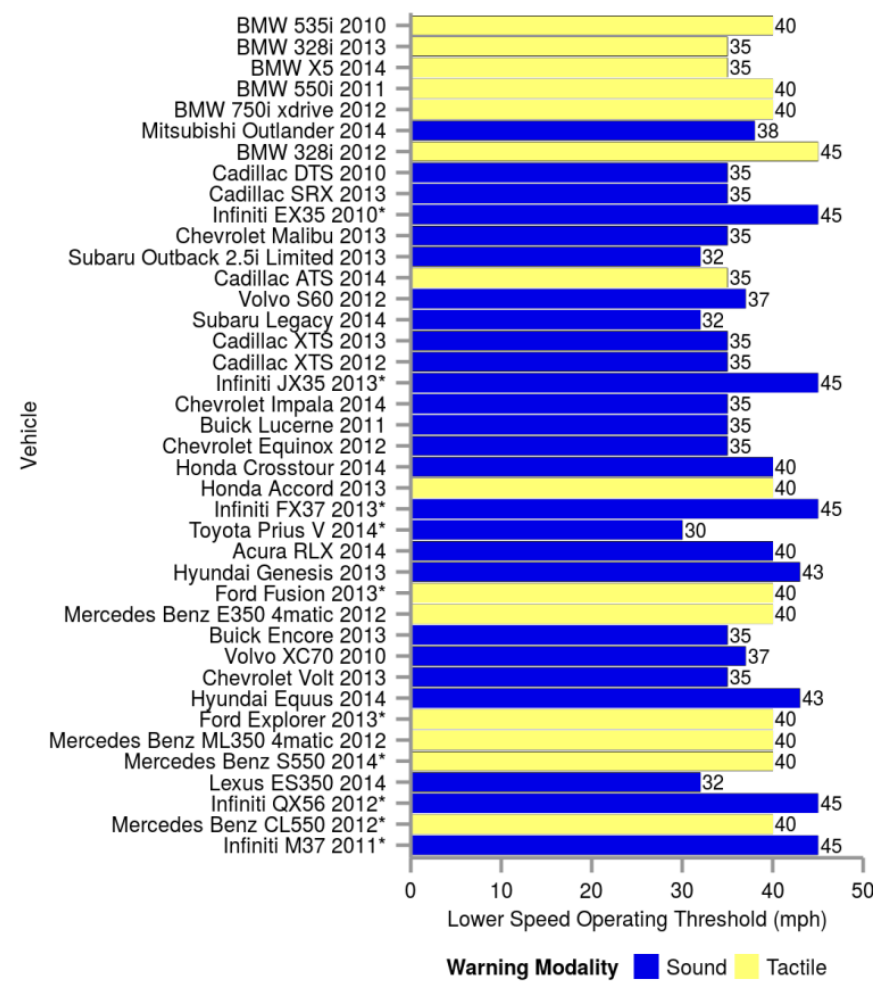

Figure 7. Lower Operating Speed Thresholds for LDW Systems. An asterisk indicates a vehicle that also had Automated Emergency Braking (AEB) equipped.

\section{Seriously Injured Drivers Prevented}

Figure 8 shows the reduction in the number of MAIS2+ drivers in rear-end collisions due to FCW. Figure 9 show the reduction in the number of MAIS2+ drivers in departure crashes due to LDW.

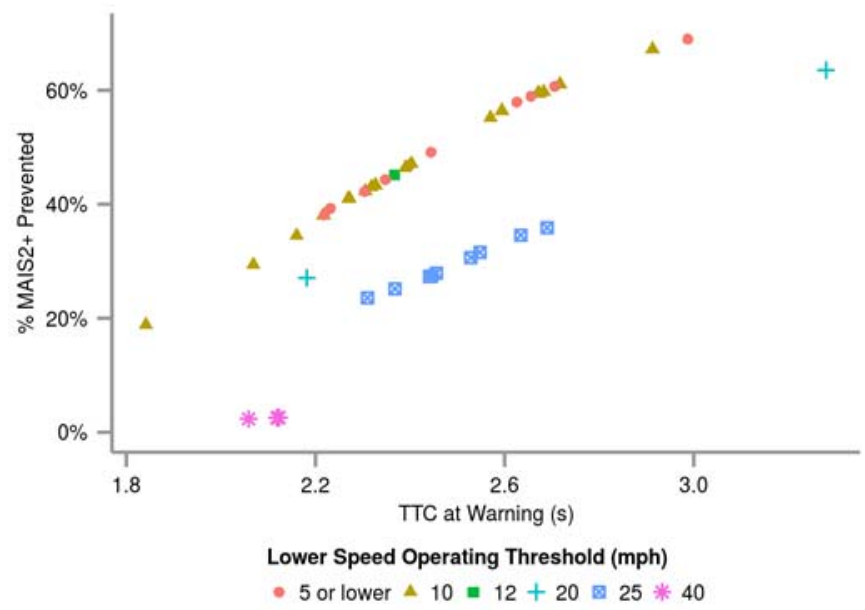


Figure 8. Reduction in the Number of MAIS2+ Drivers in Rear-end Crashes due to FCW by Warning Timing and Lower Speed Operating Threshold.

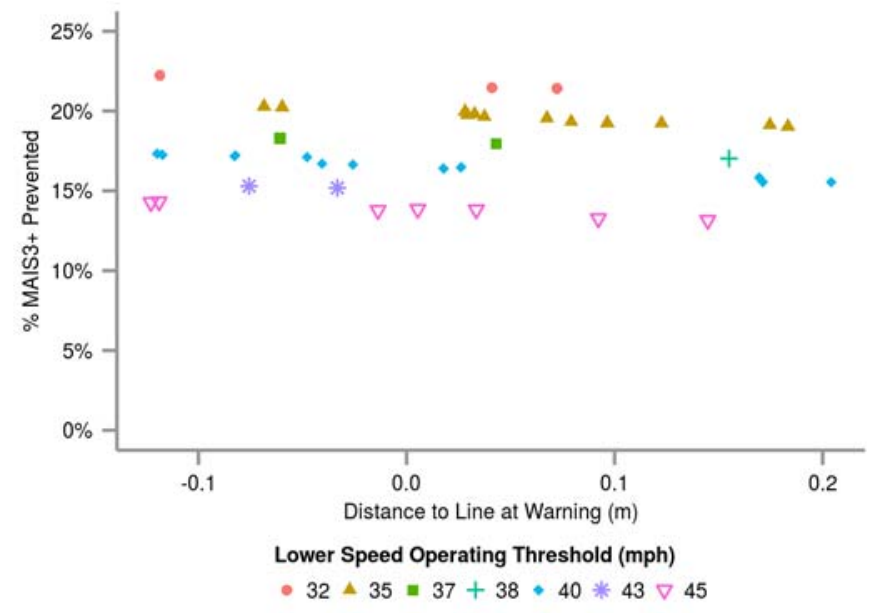

Figure 9. Reduction in the Number of MAIS3+ Drivers in Departure Crashes due to LDW by Distance to Lane Line at Warning and Lower Speed Operating Threshold. 